\title{
Dengue en un paciente anticoagulado
}

\section{Dengue in an anticoagulated patient}

Autores: Gabriel Paredes ${ }^{1}$, Daiana Correa ${ }^{2}$, Raúl Real ${ }^{3}$

Artículo recibido: 2 febrero 2014

Artículo aceptado: 25 febrero 2014

\section{Resumen}

Presentamos un caso de un paciente de sexo masculino de 73 años de edad con dengue siendo portador de una prótesis mecánica en posición aórtica y anticoagulado con acenocumarol. Ingresó con plaquetopenia por lo que se suspendió acenocumarol y fue anticoagulado con enoxaparina. Evolucionó favorablemente por lo que fue dado de alta nuevamente con acenocumarol.

Palabras claves: dengue, plaquetopenia, anticoagulación, acenocumarol, enoxaparina

\section{Abstract}

We present the case of a 73-year-old man with dengue who had a mechanical prosthesis in aortic position and was anticoagulated with acenocoumarol. As he was admitted with thrombocytopenia, acenocoumarol was suspended and anticoagulated instead with enoxaparin. The patient showed a favorable evolution and was discharged and restarted on acenocoumarol.

Keywords: dengue, thombocytopenia, anticoagulation, acenocoumarol, enoxaparin

\section{Caso clínico}

Paciente de 73 años de edad con cuadro de seis días de evolución con cefalea intensa, holocraneana, de inicio insidioso, tipo puntada. Acompañó sensación febril no graduada, con escalofríos, sin predominio de horario. Cinco días antes del ingreso se agregó náuseas y vómitos con restos de alimentos. El facultativo de cabecera lo trató ambulatoriamente y le solicitó laboratorio en el cual se constató plaquetas $20.000 / \mathrm{mm}^{3}$, motivo por el cual fue remitido al Hospital Nacional.

Antecedente de hipertensión arterial y cirugía de remplazo valvular aórtico con válvula mecánica hace 7 años, anticoagulado con acenocumarol.

${ }^{1}$ Residente de Cardiología. Hospital Juan Domingo Perón. Formosa, República Argentina

${ }^{2}$ Residente de Medicina Interna. Departamento de Medicina Interna, Hospital Nacional. Itauguá, Paraguay

${ }^{3}$ Jefe del Departamento de Medicina Interna, Hospital Nacional. Itauguá, Paraguay

Autor correspondiente:

Dr. Gabriel Paredes

Departamento de Cardiología. Hospital Juan Domingo Perón. Formosa, República Argentina

Tel: 543704512694

Correo electrónico:gabrielnparedes@hotmail.com 
Ingresó el 11 de febrero 2013 vigil, con presión arterial 100/70 mm Hg, pulso 68/min, respiraciones $20 / \mathrm{min}$, temperatura axilar $36,8^{\circ} \mathrm{C}$. En área cardiovascular tenía primer ruido cardiaco normal, clic metálico en foco aórtico, no soplos ni galope. En el resto del examen físico no se constataban signos de alarma. Serología IgM confirmó dengue. Se suspendió anticoagulación oral y administró enoxaparina $1 \mathrm{mg} / \mathrm{kp}$ cada 12 hs, vía subcutánea.

Paciente se mantuvo lúcido, con hemodinamia estable, sin signos de alarma, diuresis conservada. Laboratorio inicial mostró plaquetopenia, que mejoró en días sucesivos (tabla 1). Al normalizarse el recuento de plaquetas se reinició anticoagulación oral y fue dado de alta al $10^{\circ}$ día de enfermedad. No hubo manifestaciones hemorrágicas durante toda su estadía.

\section{TABLA 1}

Datos laboratoriales de paciente con dengue y anticoagulación

\begin{tabular}{lcccc}
\hline \multicolumn{1}{c}{ Laboratorio } & $\mathbf{1 1 - 0 2}$ & $\mathbf{1 2 - 0 2}$ & $\mathbf{1 3 - 0 2}$ & $\mathbf{1 4 - 0 2}$ \\
\hline Leucocitos & 4700 & 3000 & 2800 & 3400 \\
Neutrófilos & $46 \%$ & $56 \%$ & $51 \%$ & $47 \%$ \\
Linfo citos & $39 \%$ & $37 \%$ & $41 \%$ & $37 \%$ \\
Hemog lobina & 11 & 10,7 & 10,6 & 11,7 \\
Hematocrito & 33 & 30 & 30,8 & 33,4 \\
Plaquetas & 84000 & 85000 & 115000 & 126000 \\
Tiempo protrombina & $15 \%$ & & & $41 \%$ \\
INR & 4,4 & & & 1,8 \\
Dengue IgM & Positivo & & & \\
\hline
\end{tabular}

\section{Discusión}

El dengue es una sola enfermedad, con diferentes presentaciones clínicas y con evolución impredecible. La infección es causada por un Arbovirus, con cuatro serotipos denominados DEN 1, 2, 3 y 4. La infección por un serotipo deja inmunidad de por vida contra ese serotipo. El virus es transmitido por la picadura del mosquito hembra Aedes aegypti, el cual a su vez se infecta tras picar a una persona que está cursando el periodo virémico de la enfermedad, habitualmente los primeros 5 días desde el inicio de la fiebre. Tras un periodo de incubación de 10-12 días en el intestino del vector, el virus aparece en la saliva de la misma y a partir de ese momento puede transmitir la enfermedad a otros seres humanos. ${ }^{1}$

En los mismos, el virus produce un amplio espectro de enfermedad. La mayoría de las infecciones son asintomáticas o subclínicas pero en algunos casos pueden ser graves. Tras un periodo de incubación asintomático de 4-10 días la enfermedad comienza bruscamente y es seguida por 3 fases: febril, crítica y de recuperación. ${ }^{2}$ 
La afectación cardíaca por dengue es rara. En 1996, durante una epidemia en la India, 206 pacientes fueron evaluados y sólo uno mostró síntomas cardíacos. En la literatura, hay publicaciones de informes de casos aislados de alteraciones de la conducción auriculoventricular (ritmo de la unión y bloqueo auriculoventricular), arritmias supraventriculares y miocarditis. ${ }^{3-10}$

Dado que la plaquetopenia y la diátesis hemorrágica son complicaciones comunes del dengue, el manejo de esta infección un paciente anticoagulado es muy delicado. ${ }^{11,12}$

Un artículo publicado en el año 2007 en la Revista de la Sociedad Brasilera de Cardiología ${ }^{13}$ sugiere las siguientes recomendaciones para todos los pacientes con dengue que se encuentren anticoagulados y antiagregados:

1. Evitar la aspirina durante una semana, para reducir el riesgo de síndrome de Reye y trombocitopenia grave.

2. En pacientes con alto riesgo de trombosis, los antiplaquetarios se pueden mantener si las plaquetas son controladas periódicamente.

3. Los pacientes con alto riesgo de trombosis a corto plazo, como los pacientes sometidos a angioplastia coronaria con stent reciente (un mes para los no farmacológicos y de tres a seis meses para los farmacológicos), pacientes con prótesis valvulares mecánicas, especialmente la mitral, fibrilados, con tromboembolismo, portadores de válvula mecánica, pacientes con múltiples factores de riesgo trombótico (disfunción ventricular, ancianos hipertensos, diabéticos, enfermedad valvular cardíaca, accidente cerebrovascular previo, trombos intracavitarios): se recomienda mantener clopidogrel y AAS en los que ya los están recibiendo. Además, suspender la anticoagulación oral y sustituir por enoxaparina hasta que el INR esté por debajo del rango terapéutico. Se sugiere reintroducir la anticoagulación oral después de una semana. Es necesario monitorizar seriadamente las plaquetas y la coagulación hasta una semana. Suspender todas estas las medicaciones si el recuento de plaquetas es igual o menor de $50.000 / \mathrm{mm}^{3}$, hay hemorragias o choque cardiovascular. Se puede considerar la suspensión de clopidogrel y AAS, de conformidad con la intensidad de la reducción gradual en el número de plaquetas.

4. En los pacientes con dengue y pequeño riesgo de trombosis en el corto plazo, como con enfermedad coronaria estable, pacientes sometidos a angioplastia coronaria con stents hace más de seis meses, fibrilados sin factores de riesgo trombótico y en pacientes con prótesis valvular biológica, se recomienda suspender AAS y considerar la posibilidad de suspensión de anticoagulantes orales y clopidogrel durante una semana.

5. Los pacientes con dengue y hemorragias: suspensión inmediata de todos los antitrombóticos y anticoagulantes orales. 


\section{Referencias bibliográficas}

1. Simmons CP, Farrar JJ, Nguyen vV, Wills B. Dengue. N Engl J Med. 2012 Apr 12; 366(15): 1423-32

2. Bäck AT, Lundkvist A. Dengue viruses: an overview. Infect Ecol Epidemiol. 2013 Aug 30; 3.

3. Lee IK, Lee WH, Liu JW, Yang KD. Acute myocarditis in dengue hemorrhagic fever: a case report and review of cardiac complications in dengue-affected patients. Int J Infect Dis. 2010 Oct; 14(10): e919-22.

4. Pesaro AE, D'Amico E, Aranha LF. Dengue: cardiac manifestations and implications in antithrombotic treatment. Arq Bras Cardiol. 2007 Aug; 89(2): e12-5.

5. Lateef A, Fisher DA, Tambyah PA. Dengue and relative bradycardia. Emerg Infect Dis. 2007 Apr; 13(4): 650-1.

6. Kaushik JS, Gupta P, Rajpal S, Bhatt S. Spontaneous resolution of sinoatrial exit block and atrioventricular dissociation in a child with dengue fever. Singapore Med J. 2010 Sep; 51(9): e146-8.

7. Khongphatthallayothin A, Chotivitayatarakorn P, Somchit S, Mitprasart A, Sakolsattayadorn S, Thisyakorn C. Morbitz type I second degree AV block during recovery from dengue hemorrhagic fever. Southeast Asian J Trop Med Public Health. 2000 Dec; 31(4): 642-5.

8. Ibarrola D, Paredes G, Zarza A, Torales N, Escalada G, Cabral L. Miocarditis por dengue. A propósito de un caso. Revista CONAREC 2012; 3(05): 401-403

9. Kindermann I, Barth C, Mahfoud F, Ukena C, Lenski M, Yilmaz A et al. Update on myocarditis. J Am Coll Cardiol. 2012 Feb 28; 59(9): 779-92.

10. Real R, Rojas G, Correa D, Barrios MA, Aldama F, Pinchi L et al. Manifestaciones sistémicas del dengue. Rev Nac. (Itauguá). 2013 Dic; 5 (2): 8-17

11. MSP y BS. Dengue. Guía de Manejo Clínico 2012. Disponible en http://www.mspbs.gov.py. Visitado 1 febrero 2014

12. Gamakaranage C, Rodrigo C, Samarawickrama S, Wijayaratne D, Jayawardane M, Karunanayake P, Jayasinghe S. Dengue hemorrhagic fever and severe thrombocytopenia in a patient on mandatory anticoagulation: balancing two life threatening conditions: a case report. BMC Infect Dis. 2012 Oct 26; 12: 272

13. Pesaro AE, D'Amico E, Aranha LF. Dengue: cardiac manifestations and implications in antithrombotic treatment. Arq Bras Cardiol. 2007 Aug; 89(2): e12-5. 